\section{Sterbegeld muss versteuert werden}

Das Sterbegeld eines ärztlichen Versorgungswerks ist als Einkommen zu versteuern. Das hat der Bundesfinanzhof (BFH) aktuell entschieden (X R 13/14).

Ein niedergelassener Arzt aus BadenWürttemberg war 2008 verstorben. Als Zuschuss zu den Bestattungskosten zahlte das berufsständische Versorgungswerk seiner Ehefrau ein Sterbegeld in Höhe von $3.097 €$. Das Finanzamt betrachtete dies als steuerpflichtiges Einkommen, wegen der stufenweisen Umstellung auf die nachgelagerte Besteuerung von Renten jedoch erst bei der Auszahlung, hier mit einem Anteil von $56 \%(1.734 €)$. Die hiergegen gerichtete Klage der Witwe hatte vor dem Finanzgericht in Stuttgart noch Erfolg. Der BFH hob dieses Urteil nun aber auf und wies die Klage ab. Zur Begründung verwies er auf sein Grundsatzurteil vom Oktober 2013. Danach sind Kapitalleistungen berufsständischer Versorgungswerke seit 2005 zu versteuern. Sie werden ebenso wie monatliche Versorgungsleistungen von der gegenwärtigen Umstellung auf die nachgelagerte Rentenbesteuerung erfasst.

Nach dem neuen Urteil gilt dies für "sämtliche Auszahlungen aus der Basisversorgung", soweit sie bereits von der nachgelagerten Besteuerung erfasst sind. Der Zweck des Sterbegeldes, eine Hilfe für die Beerdigungskosten zu geben, ändere daran nichts, denn eine entsprechende Zweckbindung bestehe rechtlich gesehen nicht. Weiter entschied der BFH, dass das Sterbegeld dem regulären Steuersatz unterliegt. Die Vergünstigungen für Kapitalleistungen, die Versicherungen anstelle einer Rente auszahlen, seien hier nicht anwendbar.

Martin Wortmann

\section{Mustervorlage für Hygieneplan}

Das Kompetenzzentrum Hygiene und Medizinprodukte der KVen und der KBV $(\mathrm{CoC})$ stellt eine Mustervorlage für den Hygieneplan zum kostenlosen Download im Internet bereit: goo.gl/Z6kDae. $\mathrm{CoC}$ will den Praxen ein Unterstützungsund Serviceangebot für die Erstellung des praxisinternen Hygieneplans an die Hand zu geben. In der 83 Seiten umfassenden Mustervorlage werden fachübergreifend hygienerelevante Abläufe einer Praxis detailliert dargestellt. Die Regelungen beschreiben allgemeine, aber auch spezielle, Hygienemaßnahmen.

Matthias Wallenfels zu Gesundheitsthemen auch für Kurgäste und die Einbindung von Freiwilligen, so Vorstand Marcus Jünemann.

Ein anderes Beispiel: Möglichst niedrigschwellig will das Ärztenetz BillstedtHorn e. V. im Rahmen des Projektes Invest die Versorgung in dem sozial schwachen Hamburger Stadtteil organisieren. Dazu hat es 2016 mit Unterstützung durch die Optimedis AG gemeinsam mit der SKH Stadtteilklinik Hamburg GmbH und dem NAV Virchowbund die Gesellschaft Gesundheit für Billstedt/ Horn gegründet. Das Modell soll die rund 120 Ärzte vor Ort entlasten. Allerdings braucht das Netz noch deutlich mehr Beteiligung, sagt Netzvorstand Dr. Gerd Fass. Das Projekt wird mit 6,3 Millionen $€$ aus dem Innovationsfonds gefördert.

Angela Miss/beck

\title{
„Es klappt ja doch mit Familie!"
}

\author{
Beruf und Familie - das funktioniert auch in eigener Praxis. Vielleicht sogar \\ besser als im Krankenhaus. Diese Erkenntnis konnten junge Ärzte vom „Tag \\ der Chancen“ in Thüringen im März mitnehmen.
}

$D^{\circ}$ er Frauenanteil bei in Einzelpraxis niedergelassenen Ärzten in Thüringen liegt bei $60 \%$ und ist damit überproportional hoch. Bei Berufsausübungsgemeinschaften (BAG) liegt er mit $52 \%$ etwas niedriger, aber immer noch über $50 \%$. Diese Zahlen zeigen, dass das alte Vorurteil, die Niederlassung bringe eine so hohe Arbeitsbelastung mit sich, dass Beruf und Familie nur schwer miteinander zu vereinbaren seien, nicht mehr mit der Realität zusammenpasst, so Peter Hedt, Niederlassungsberater der KV Thüringen und Leiter eines Workshops am 4. März 2017 in Weimar, der gemeinsam von Kassen(zahn)ärztlicher Vereinigung, Landes(zahn)ärztekammer und Deutscher Apotheker- und Ärztebank (apoBank) veranstaltet worden ist.

„Es ist nicht nur wider Erwarten doch machbar, Beruf und Familie in der Niederlassung miteinander zu vereinbaren, sondern es ist sogar leichter möglich als in der Anstellung in der Klinik“, ergänzt Bernhard Koelmer, Filialleiter der apoBank in Erfurt. Die jungen Ärzte seien sich zwar bewusst, dass sie viel arbeiten müssen, „aber sie können es selbst gestalten“. Das fange schon damit an, dass Kinder auch mal mit in die Praxis genommen werden könnten - was auf Station kaum möglich ist. Vorteilhaft seien auch die _flexible Gestaltung der Arbeitszeit, - Auswahl der passenden Mitarbeiter, um die Belastung zu senken,
-Unabhängigkeit von unternehmerischen Entscheidungen anderer,

_Entscheidungsfreiheit, ob Verantwortung und Risiko in einer Kooperation geteilt werden soll, oder ob die Einzelpraxis präferiert wird.

Durch die Gesetzgebung der vergangenen Jahre hätten sich die Gestaltungsmöglichkeiten ärztlicher Selbstständigkeit entscheidend erweitert, betont Hedt, „und wir kommen unseren Mitgliedern so weit wie möglich entgegen". Das schon lange mögliche Jobsharing oder auch eine Teilzulassung schafften mehr Zeit für das Privatleben. So könne eine BAG beispielsweise gemeinsam geführt werden - einer der Partner arbeitet am Vormittag, der andere am Nachmittag.

Zunehmend genutzt wird laut Hedt auch die Beschäftigung eines Sicherstellungsassistenten - je Kind ist das drei Jahre lang möglich. Aktuell gebe es 31 Sicherstellungsassistenten in Thüringen, 16 davon hätten die Genehmigung 2016 erhalten, und zwölf seien bereits in den ersten beiden Monaten 2017 dazugekommen. Und auch bei Beschäftigung eines Sicherstellungsassistenten könne der Inhaber (kürzer) weiterarbeiten, um den Kontakt zur Praxis nicht zu verlieren. „Mögliche höhere finanzielle Risiken durch kürzere Arbeitszeiten sind kein Thema für uns", so Koelmer. Die jungen Ärzte gingen „realistisch in die Planung" und passten Investitionen und andere Kosten an die Einkommensmöglichkeiten an. Hauke Gerlof 